\title{
Términos teóricos y teorías híbridas de la referencia
}

\author{
Theoretical Terms and Hybrid Theories of Reference
}

\author{
Dalila Serebrinsky*; Bruno Borge** \\ *Universidad de Buenos Aires, Argentina \\ dalila.r.ser@gmail.com \\ **Universidad de Buenos Aires / CONICET, Argentina \\ brunojborge@gmail.com
}

\begin{abstract}
Resumen
El descriptivismo y la teoría causal de la referencia fallan en dar cuenta del significado de los términos teóricos de un modo coherente con el realismo científico. Ante ese problema, se han desarrollado teorías híbridas de la referencia que combinan rasgos de ambas y que pretenden recoger las ventajas de cada una. En este trabajo analizamos críticamente dos estrategias para articular teorías híbridas de la referencia ante el problema del significado de los términos teóricos, ejemplificadas por las propuestas de Psillos y Kitcher. Argumentamos que ninguna de estas estrategias tiene éxito en articular los elementos descriptivos y causales en una genuina teoría híbrida de la referencia que satisfaga las pretensiones del realismo científico.
\end{abstract}

Palabras clave: descriptivismo causal, referencia directa, teoría causal de la referencia, realismo científico, cambio científico.

\section{Abstract}

Both descriptivism and the causal theory of reference fail to account for the meaning of theoretical terms in a way consistent with scientific realism. Faced with this problem, hybrid theories of reference have been developed. They combine features of both descriptivism and the causal theory and seek to capture the advantages of each. In this work, we critically analyze two strategies to articulate hybrid theories

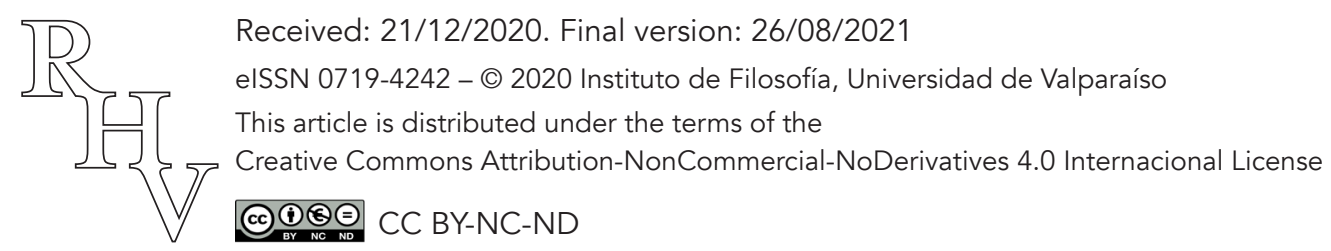


of reference in the face of the problem of the meaning of theoretical terms. They are exemplified by the proposals of Psillos and Kitcher. We argue that neither of these strategies is successful in articulating the descriptive and causal elements in a genuine hybrid theory of reference that satisfies the standards of scientific realism.

Keywords: causal descriptivism, direct reference, causal theory of reference, scientific realism, scientific change.

\section{Introducción}

El realismo científico sostiene que nuestras teorías científicas actuales son aproximadamente verdaderas. Esta posición se compromete, por lo tanto, con la existencia de referentes para los términos que aparecen en estas teorías, y esto incluye, por supuesto, los términos teóricos. El realista científico debe explicar, entonces, cómo los términos teóricos se vinculan con sus referentes ${ }^{1}$. No obstante, además del compromiso con la verdad (aproximada) de las teorías vigentes, el realismo adopta un compromiso con el carácter continuo y acumulativo del conocimiento científico. La verdad (aproximada) de las teorías que hoy aceptamos descansa en una dinámica histórica en la que el conocimiento científico logra incrementarse de modo lineal a lo largo del cambio teórico. Es así que los términos teóricos representan un desafío doble para las teorías de la referencia, ya que cualquier teoría que pretenda explicar la relación entre estos términos y sus referentes en clave realista debe cumplir con las siguientes condiciones:

1. La referencia de los términos teóricos debe depender, al menos en parte, de las teorías en las que estos aparecen.

2. La referencia debe poder rastrearse transteóricamente, es decir, se debe poder dar cuenta de cómo los términos refieren a la misma entidad, aunque aparezcan en teorías diferentes.

Actualmente, existen dos teorías que compiten por explicar la referencia de los nombres propios y términos de clase natural: el descriptivismo y la teoría causal de la referencia. Pero ninguna de ellas es capaz de satisfacer ambas condiciones. La teoría descriptivista cumple con la primera, pero es incapaz de abordar la segunda. La teoría causal de la referencia, en cambio, puede explicar que los términos sean transteóricos, pero no es capaz de dar cuenta del papel de la teoría científica en la fijación del referente.

\footnotetext{
${ }^{1}$ Esta caracterización estándar del realismo científico no es, desde ya, universal. Las posiciones realistas son hoy en día tan diversas que resulta complejo encontrar una definición unívoca. En particular, formas de realismo científico selectivo como el realismo estructural (Worrall 1989) pueden ameritar enfoques alternativos al de las teorías de la referencia abordadas en este trabajo. De ese modo, nuestros argumentos se dirigen a las versiones del realismo alcanzadas por esta caracterización. Para más detalles sobre variantes del realismo científico véase Borge (2015).
}

Revista de Humanidades de Valparaíso, 2021, No 17, 169-191 
Ante la insuficiencia de ambas teorías en su versión "pura", se han propuesto teorías híbridas de la referencia que combinan rasgos de ambas y que pretenden recoger las ventajas de cada una. No obstante, hay más de una estrategia posible a la hora formular una teoría híbrida. Distintas versiones de la teoría híbrida denominada descriptivismo causal proponen que la fijación del referente de un término teórico depende de un elemento descriptivista y, a la vez, de un elemento causal, de manera que ambos son necesarios para atribuir referencia al término. Otras propuestas, en cambio, no buscan amalgamar los componentes causal y descriptivo en un único mecanismo de fijación de la referencia de los términos teóricos, sino distinguir instancias particulares de términos que fijan su referencia autónomamente, alternando modos de referencia descriptivos y causales.

El objetivo de este trabajo es mostrar que ninguna de estas estrategias tiene éxito en articular los elementos descriptivos y causales en una genuina teoría híbrida de la referencia que permita satisfacer conjuntamente los requisitos 1 y 2 , y así, de acuerdo a la pretensión realista, ser capaz de rastrear la referencia de los términos que refieren a inobservables a lo largo del cambio teórico. Para eso, analizaremos las teorías híbridas propuestas por Psillos y por Kitcher, que toman la primera y la segunda de estas estrategias, respectivamente, y mostraremos cómo cada una falla en explicar la referencia de los términos teóricos de acuerdo a estos requisitos ${ }^{2}$.

En la segunda sección, presentaremos las teorías de la referencia disponibles. En la tercera sección, expondremos el descriptivismo causal tal como fuera propuesto por Psillos. Luego, en una segunda subsección, presentaremos argumentos para mostrar que el descriptivismo causal funciona como cualquier teoría descriptivista, sin otorgar al elemento causal ningún papel real en la determinación de la referencia. En una tercera subsección, mostraremos cómo, incluso si se involucrara un elemento causal en la fijación del referente, la teoría no podría ser híbrida, ya que entonces no se podría atribuir un papel al elemento descriptivista y resultaría una teoría puramente causal. En la cuarta sección presentamos críticamente un intento de formular una teoría híbrida que ilustra la segunda estrategia mencionada arriba: la propuesta de Kitcher (1993). Sostenemos que distintas dificultades conceptuales socavan su pretensión de articular satisfactoriamente elementos descriptivos y causales, por lo que no constituirse como una genuina teoría híbrida de la referencia. Finalmente, en la última sección, presentaremos las conclusiones de este trabajo.

\footnotetext{
${ }^{2}$ Por supuesto, las dos teorías consideradas aquí no agotan la totalidad de las teorías híbridas de la referencia. Sería imposible analizar todas en este artículo. Aunque sospechamos que los resultados de este trabajo pueden alcanzar a otras teorías híbridas, no descartamos que se necesiten argumentos adicionales para mostrar la inviabilidad de otras propuestas, como las de Devitt y Sterelny (1987), Enç (1976) y Kroon (1975), por mencionar sólo algunas. Para un análisis de las estrategias tomadas por las dos últimas, véase Borge y Serebrinsky (2022, en prensa).
}

Revista de Humanidades de Valparaíso, 2021, No 17, 169-191 


\section{Teorías de la referencia}

\subsection{Las teorías descriptivistas}

Las teorías descriptivistas proponen que el referente de un nombre está determinado por una descripción o conjunto de descripciones asociado a ese término. Para el descriptivismo clásico (v. g. Frege 1892; Russell 1911) lo expresado por un nombre equivale a lo expresado por una descripción definida asociada al término. La referencia de un nombre es el objeto que satisface esa descripción definida asociada. Usar un nombre propio de manera competente es, entonces, conocer la descripción definida correcta y asociarla con ese nombre. Otras teorías descriptivistas posteriores sugieren, en cambio, abandonar la exigencia de que el término esté asociado a una descripción definida, sino que proponen que la referencia esté determinada por un conjunto de descripciones que pueden ser indefinidas, de manera que el referente sea el objeto que cumple con la mayoría de estas descripciones (Searle 1958). Para estas teorías, también, los hablantes pueden referir mediante el nombre en virtud de su conocimiento de las descripciones asociadas correspondientes.

Las teorías descriptivistas, si bien fueron concebidas para explicar la referencia de los nombres propios, fueron extendidas a otros tipos de términos. Fueron aplicadas, por ejemplo, a términos de clases naturales (v.g. Putnam 1975). Es entonces una de las propuestas disponibles para explicar la referencia de los términos teóricos, ya que estos pretenden designar clases naturales inobservables. Sin embargo, estas teorías no son capaces de satisfacer una de las condiciones que mencionamos en la sección anterior. Una teoría descriptivista no puede dar cuenta del hecho de que los términos puedan rastrear su referente transteóricamente. Si el referente de un término está determinado por la descripción (o el conjunto de descripciones) asociada al término, entonces si un término aparece asociado a distintas descripciones en distintas teorías, no puede referir a lo mismo en todos sus usos. Un cambio de teoría tal llevará necesariamente a un cambio de referente, ya que las descripciones que hace cada teoría no podrán seleccionar la misma entidad. Sin embargo, es claro que en muchos casos los científicos describen cierta entidad de manera diferente, y aun así aceptan que están hablando de lo mismo.

\subsection{La teoría causal de la referencia}

Para la teoría causal de la referencia, de un nombre está determinada por la relación causal que existe entre los usos de ese nombre y la entidad que es su referente. Un término adquiere su referente, según esta propuesta, en un acto de bautismo donde un hablante usa por primera vez el término para referirse a una entidad. En ese caso, ese uso del término es causado por la entidad misma a la cual refiere, pero otros usos posteriores no necesitan ser causados "directamente" por esa entidad, sino que basta con que estén conectados causalmente a otros usos, alguno de los cuales sí está causado por la entidad en cuestión

Revista de Humanidades de Valparaíso, 2021, No 17, 169-191

(c) $(1)(9)$ CC BY-NC-ND 
(Kripke 1972, 302). Se dice entonces que debe existir una cadena histórico-causal que conecte los usos de cada hablante con el primer uso ocurrido en el acto bautismal. Como vemos, esta propuesta no exige la mediación de descripciones o conceptos. Solo se necesita que el hablante esté conectado de la manera correcta con la entidad, ya sea en el acto en el que se fija por primera vez la referencia o al heredar posteriormente su uso de otros hablantes.

La teoría causal de la referencia fue introducida para nombres propios pero fue también concebida para explicar la referencia de los términos de clase natural. Putnam (1975) propuso que estos términos también adquieren su referencia causalmente, por medio de un acto de ostensión que fija la referencia del término, que en este caso no es el objeto o la muestra presente, sino todo objeto o sustancia que pertenezca a la clase del objeto o muestra señalado. En el caso de los términos de magnitudes físicas como 'temperatura' o 'carga eléctrica', por ejemplo, el uso del término está conectado por medio de una cadena causal a la situación en la que se dio por primera vez una descripción de la electricidad como la magnitud física responsable de ciertos efectos (Putnam 1973, 200). Putnam denomina evento introductorio a la situación en la que se introduce el término en el lenguaje. Los usos posteriores del término remitirán causalmente al evento introductorio. Así, por ejemplo, una vez que el término 'electricidad' ha sido introducido en el vocabulario de una persona, ya sea directamente en el evento introductorio o a través de una cadena causal que conecta la palabra a dicho evento, la referencia queda automáticamente fijada: la electricidad es la magnitud física responsable de tales y tales efectos. No obstante, si diferentes hablantes asocian a la magnitud descripciones diferentes refieren a la misma entidad, esto es, a aquella responsable de los efectos en cuestión, independientemente de que la información asociada en la descripción sea correcta o equivocada. Es por ello que una explicación causal tiene problemas para dar cuenta de los términos teóricos que designan clases inobservables de un modo que satisfaga las pretensiones realistas. No está claro, en estos casos, cómo funcionaría un acto ostensivo para seleccionar el referente y anclar la referencia a lo largo del cambio teórico, ni cuál podría ser la relación causal entre la entidad y el hablante que permitiría un acto de bautismo. Por otro lado, una teoría causal no puede explicar el fallo referencial: si un término refiere a aquello que causó su primer uso, no puede ser vacío. Los casos de fallo referencial, sin embargo, abundan en la historia de la ciencia. Además, esta teoría no cumple la segunda condición que mencionamos en la primera parte: si la relación referencial está dada sólo por la relación causal entre el referente y los usos del término, entonces no se explica cómo intervienen las teorías científicas que contienen términos teóricos en la determinación de la referencia de esos términos. Para un defensor de la teoría causal de la referencia, el término referirá a aquella entidad causalmente responsable de sus usos, independientemente de cómo la teoría la describa. 


\section{Psillos: el descriptivismo causal}

Ante la incapacidad de cada una de las teorías presentadas para satisfacer las dos condiciones que requiere una teoría de la referencia de los términos teóricos, Psillos (1999; 2012) ofrece una tercera alternativa: el descriptivismo causal (DC). Esta teoría es propuesta como un híbrido entre el descriptivismo y la teoría causal, que reúne las ventajas de ambas. La referencia de los términos teóricos, para Psillos, debe ser explicada apelando a un mecanismo que incluya un elemento causal y, a la vez, un elemento descriptivista. La relación de referencia en estos casos podría caracterizarse de la siguiente manera (2012, 222):

$$
\begin{aligned}
& \mathrm{R}(x)=x \text { causa el fenómeno } \Phi y \mathrm{D}(x) . \\
& \text { Un término } t \text { refiere a } x \text { si y sólo si se da } \mathrm{R}(x) .
\end{aligned}
$$

Entonces, $x$ debe cumplir una condición causal, ser la causa del fenómeno indicado, y una condición descriptivista, satisfacer la descripción definida o conjunto de descripciones D. El elemento D, además, es caracterizado como una descripción causal, es decir, que incluye aquellas propiedades que son relevantes para explicar el hecho de que la entidad causa el fenómeno $\Phi$. Una entidad que sea la causa de $\Phi$ pero que no satisfaga $D$ no será el referente de $t$, y tampoco lo será una entidad de la cual $\mathrm{D}$ sea verdadera pero que no cause el fenómeno $\Phi$.

\section{1 ¿En qué sentido el descriptivismo causal es causal?}

El DC pretende ser una teoría híbrida que integra lo mejor de las teorías de la referencia disponibles. Sin embargo, si bien el elemento descriptivista es indiscutible, no está claro en qué sentido esta teoría es una teoría causal. El componente que el autor pretende introducir como garante de la relación causal es, justamente, la relación causal que debe existir entre la entidad a la que se pretende referir y el fenómeno que se observa. Sin embargo, si bien efectivamente esta es una relación causal, no es el tipo de relación causal que una teoría causal de la referencia requiere. Recordemos que la teoría causal de la referencia exige que exista una relación histórico-causal que conecte a cada hablante con un acto de bautismo en el cual, a su vez, existió una relación causal entre el referente y el/los hablante/s que participaron de él. Ninguna de estas relaciones está expresada en la formulación del elemento causal de la teoría de Psillos.

Por otro lado, no está claro si esta relación debe ser conocida por el hablante. Si es así, entonces ese componente constituye una descripción, es decir, una expresión que atribuye ciertas propiedades a una entidad, y no estaría claro por qué hay que proponerla como una condición aparte del conjunto de descripciones D. Si el componente causal no es una descripción que el hablante tenga que conocer para referir exitosamente, en cambio, no está claro cómo interviene en la relación de referencia. 
La imposibilidad de esta condición para participar en un mecanismo causal de fijación de la referencia no está dada por el hecho de que sea una descripción causal. El uso de descripciones (causales o no) no es en sí mismo un problema para una teoría causal de la referencia. De hecho, para las teorías de este tipo, hay dos maneras en las que puede seleccionarse el referente en el acto bautismal: a través de una descripción o por medio de un acto ostensivo. Cuando se trata de entidades inobservables, es poco claro en qué consistiría la selección ostensiva de la entidad que pretendemos que sea el referente de nuestro término. Parece viable entonces proponer que el bautismo en estos casos se hace por medio de una descripción. En la propuesta de Psillos, sin embargo, la descripción causal cumple otro rol. No se trata de una entidad lingüística que un primer hablante usa para fijar la referencia en el acto bautismal y que después es abandonada para dejar la cadena histórico-causal entre los usos de los hablantes como único garante de la relación referencial. Al incluir la descripción causal como condición de la relación de referencia, está proponiendo que estas descripciones contribuyen a fijar la referencia en todos los usos de todos los hablantes, y no sólo en el bautismo. La descripción causal estaría siendo usada de la manera en que se usa en una teoría descriptivista de la referencia, es decir, como una descripción que selecciona de forma única al referente y que necesita estar asociada al término para que éste refiera. Este elemento causal, sin embargo, no parece estar funcionando ni siquiera como una descripción causal. El papel explicativo que le da Psillos a este elemento parece ser ontológico, no semántico, ya que se trata de la relación causal entre la entidad y algún fenómeno, y no de la relación causal entre la entidad y los usos de los hablantes. Si es así, tenemos aun más razones para afirmar que esta propuesta semántica es nada más que un descriptivismo.

Tenemos entonces razones para afirmar que la supuesta teoría híbrida de Psillos no es más que otra teoría descriptivista y es, por lo tanto, objeto de las críticas habituales hacia este tipo de teorías. Una de ellas es que es incapaz de dar cuenta del modo en que un término mantiene su relación de referencia con la misma entidad a través de diferentes teorías científicas, o por lo menos lo es si no logra dar con la caracterización adecuada de D y, como veremos, no lo hace. Sin embargo, aun si suponemos que la descripción causal que Psillos presenta es un auténtico mecanismo causal de fijación de la referencia, podemos pensar que no cumple esa función en la explicación de la relación referencial que el mismo autor propone.

\subsection{Dos versiones del descriptivismo causal}

Como vimos, el DC sostiene que la referencia de un término $t$ a una entidad $x$ está dada por una relación R. Para que se dé $\mathrm{R}$, es necesario y suficiente que se den dos condiciones: la satisfacción por parte de $x$ de una descripción causal y la satisfacción por parte de $x$ de un conjunto de descripciones D. Estas condiciones pretenden ser una condición causal y una descriptivista, respectivamente. Es decir, que la primera condición debería funcionar 
como lo hace la relación entre referente y hablante en la teoría causal de la referencia y la segunda condición debería funcionar como lo hacen las descripciones en una teoría descriptivista. Recordemos que, en ésta última, las descripciones logran fijar la referencia por estar asociadas al término, de manera que, no sólo son verdaderas del objeto al que pretende referir, sino que son conocidas por todos los hablantes capaces de usar ese término. Psillos ofrece dos versiones de su DC que se diferencian entre sí por la caracterización que cada una hace de este elemento descriptivista.

\subsubsection{Propiedades esenciales/constitutivas de clase}

Las propiedades esenciales son aquellas que la entidad, si existe, necesariamente tiene. Psillos (1999) caracteriza las propiedades constitutivas de una clase natural como las propiedades esenciales de esa clase y su primera versión del DC sostiene que son estas propiedades las que deben estar incluidas en D. Una descripción de la entidad en términos de sus propiedades esenciales sería una condición necesaria para referir exitosamente a ella. La descripción $\mathrm{D}$ es el elemento descriptivista de la relación $\mathrm{R}$ y requiere que cada hablante conozca esta descripción y la asocie al término $t$. Pero recordemos que una explicación descriptivista no es suficiente, ya que no puede dar cuenta de la continuidad referencial a través de diferentes teorías. para que se dé $\mathrm{R}$ es necesario, además, que se cumpla el elemento causal, es decir, que $x$ sea la causa del fenómeno $\Phi$. La satisfacción de ambas condiciones garantizaría la referencia de $t$ a $x$.

Pero veamos cómo funciona esta explicación de la referencia cuando la descripción D consiste en las propiedades esenciales de $x$. Si se cumple la condición descriptivista tanto como la condición causal, entonces el término teórico $t$ refiere a $x$. Si la entidad $x$ causa el fenómeno $\Phi$ pero no la descripción $\mathrm{D}$, entonces $t$ no refiere. Si $x$ satisface la descripción $\mathrm{D}$, pero no causa $\Phi$, ¿falla la referencia? No está claro cómo podría ser así. Si D es una genuina descripción verdadera de la entidad $x$ que la selecciona por medio de sus propiedades esenciales, entonces no importa si la entidad de hecho causa o no el fenómeno en cuestión, ya que, entonces la descripción de la entidad no podría cambiar de una teoría a la otra, y el problema de la continuidad referencial no sería tal. Por otro lado, si la descripción D sí cambiara entre teorías, al tratarse de las propiedades esenciales de la entidad, necesariamente cambiaría el referente, más allá de que la entidad cause el fenómeno o no. En cualquier caso, el elemento causal no parece cumplir ninguna función a la hora de explicar la referencia de $t$.

Si D funciona como un genuino elemento descriptivista, entonces D debe ser tal que cada hablante la conozca y la asocie con el término. Si fuera así, no existirían hablantes que usen exitosamente el término en cuestión sin conocer la descripción $\mathrm{D}$, por lo que no podría objetarse esta explicación de la referencia por el argumento semántico. Además, en el caso de que la descripción funcionara así, si una entidad $e$ tuviera una propiedad esencial $\mathrm{F}$, ésta debería, por exigencia de esta misma teoría causal descriptivista, estar incluida

Revista de Humanidades de Valparaíso, 2021, No 17, 169-191

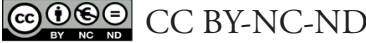


en $\mathrm{D}$, la cual, como dijimos, es conocida por todos los hablantes que usan el término $t$ para referir a $e$. Entonces, una proposición expresada por algo del tipo " $e$ es F" debería ser conocida a priori por cada uno de estos hablantes. Tampoco funcionaría entonces el argumento epistémico en contra de una explicación basada exclusivamente en el componente descriptivista. Finalmente, si D incluye todas las propiedades esenciales de la entidad y nada más que sus propiedades esenciales, y si las propiedades esenciales son aquellas que la entidad, si existe, necesariamente tiene, entonces a través de esa descripción se refiere a la misma entidad en todos los mundos posibles en los que esta entidad existe. La descripción $\mathrm{D}$, por sí misma, designa rígidamente a la entidad. Una explicación de la referencia por medio de la descripción D no sería objeto del argumento modal. Una descripción que incluya todas las propiedades esenciales de una entidad $e$ y nada más que sus propiedades esenciales sería lo suficientemente poderosa como para explicar por sí misma la referencia de un término a $e$. Si un término teórico está asociado a una descripción de este tipo, entonces, no se necesita nada más para explicar su referencia.

Una teoría que se apoya en una descripción de las propiedades esenciales de las entidades inobservables para explicar la referencia de los términos teóricos presenta, sin embargo, un problema: los términos teóricos simplemente no funcionan así. Es un hecho que hay hablantes que usan términos teóricos exitosamente sin conocer las descripciones que se supone que están asociadas a ellos. De hecho, la mayoría de los hablantes usan los términos teóricos de esa manera. Al introducir el término, en general, no se conocen las propiedades esenciales de la entidad a la que pretendemos referir. Justamente, la tarea de la ciencia es, entre otras cosas, descubrir esas propiedades. Pero no por eso pensamos que, hasta completar esa tarea, los científicos son incapaces de referir a las entidades acerca de las cuales tratan sus teorías. Esta dificultad de la formulación de la descripción D (que se parece a la dificultad señalada a los descriptivistas por el argumento semántico) no puede ser salvada por el elemento causal en una teoría híbrida como la de Psillos, ya que la relación de referencia que propone este autor exige que se cumpla tanto la condición causal como la condición descriptivista, y vemos que ésta última no se cumple si consiste en una descripción de la entidad por medio de sus propiedades esenciales. Recordemos que, para que la condición descriptivista sea satisfecha, no sólo la descripción debe ser verdadera (y, si esa descripción incluye nada más que propiedades esenciales de la entidad, es necesariamente verdadera), sino que debe estar asociada al término y ser conocida por todos los hablantes que usen exitosamente ese término. Es claro, entonces, que esta versión del DC no explica la referencia de los términos teóricos, ya que la condición descriptivista no se cumple, y eso es suficiente, dentro de esta teoría, para que falle la referencia. Pero si la condición descriptivista fuera satisfecha, entonces eso sería suficiente para dar cuenta de la referencia de los términos, sin la intervención de ningún elemento causal. 


\subsubsection{Propiedades estables contingentes}

Psillos (2012) advierte la dificultad de usar propiedades esenciales para caracterizar D y propone una segunda versión de su DC, en la cual el elemento descriptivista incluye, ya no propiedades que la entidad necesariamente tiene, sino propiedades estables contingentes. Éstas serían propiedades estables de esta clase de entidades que, sin ser constitutivas de esa clase, explicarían su rol causal.

Cuando la descripción D está constituida por las propiedades estables contingentes de la entidad, no es necesario que el referente cambie cuando cambia la descripción. Puede haber dos descripciones D y D' que refieran a la misma entidad, aunque esto último no sea conocido por los hablantes que usan los términos asociados a D y D'. Como cada descripción debería asociarse a un término y determinar su referencia, los casos en los que se asocian descripciones diferentes son aquellos en los cuales se supone que el referente es diferente. Puede descubrirse más tarde, sin embargo, que ambos términos con sus respectivas descripciones refieren al mismo objeto. Este parece ser un escenario bastante común y del que se pueden citar muchos ejemplos. Tal vez el más paradigmático sea el ejemplo fregeano de Héspero y Fósforo, con las descripciones asociadas a cada uno de estos términos. Héspero refiere a Venus en virtud de estar asociado a la descripción "la estrella vespertina" y Fósforo refiere al mismo objeto, Venus, pero por estar asociado a otra descripción, "la estrella matutina". El objeto, Venus, satisface ambas descripciones. Entonces, parece que hay un buen pronóstico acerca de esta versión del descriptivismo causal: permitiría, en principio, la continuidad referencial de un término, incluso si las teorías en las que aparece describen su referente de manera distinta. Este pronóstico se cumplirá en tanto las descripciones de las diferentes teorías sean compatibles entre sí, y eso casi nunca sucede. En cualquier caso, aún no está claro qué papel cumple la condición causal en este relato.

Podría pensarse que nos enteramos de que distintas descripciones refieren al mismo objeto cuando tenemos algún tipo de relación no conceptual con el objeto. No está claro que la relación no conceptual requerida deba ser una relación causal. Pero supongamos que lo fuera. En este tipo de situación, entonces, se cumplen las dos condiciones que Psillos exige para que se dé la relación de referencia. Sin embargo, no puede decirse que cumplan las funciones que Psillos les atribuye, esto es, la función de garantizar la referencia. Héspero, como la estrella vespertina, y Fósforo, como la estrella matutina, ya referían a Venus antes de que los hablantes sepan que en realidad nombran al mismo objeto. Los términos asociados a descripciones diferentes que, no obstante, tienen el mismo referente pueden referir y ser usados exitosamente por los hablantes en virtud de, solamente, su conocimiento de esas descripciones. El hecho de que se pueda reidentificar el referente seleccionado por dos descripciones diferentes por un contacto causal posterior, en todo caso, sólo muestra que ese contacto causal no era necesario para que los términos refieran en primer lugar. Una vez más, el elemento causal propuesto por Psillos no parece cumplir ninguna función para garantizar la referencia.

Revista de Humanidades de Valparaíso, 2021, No 17, 169-191 
Podría decirse, sin embargo, que el elemento causal cumple la función de garantizar que haya un referente. Después de todo, puede haber, según el descriptivismo, un término asociado a una descripción no satisfecha, pero no puede haber, desde la teoría causal de la referencia, un nombre sin un objeto que cause su uso. Pero si la descripción es satisfecha, entonces existe un objeto que la satisface y, por lo tanto, un referente para nuestro término. Y si la descripción no se satisface, entonces, según el DC, no podremos decir que hay referente, haya o no un objeto que cumpla con la condición causal, ya que esta teoría requiere que se cumplan tanto la condición causal como la descriptivista para que haya referencia. Por lo mismo, no puede alegarse que el elemento causal garantiza la continuidad referencial en aquellos casos en los que hay dos o más descripciones y éstas son incompatibles entre sí. En el ejemplo presentado más arriba, el objeto satisface ambas descripciones. Pero supongamos que tenemos una teoría $\mathrm{T}$ en la que aparece el término $t$ para referir a la entidad $e$, caracterizada con la descripción $\mathrm{D}$. Y que tenemos, también, una teoría T' en la que aparece el término $t$ para referir a $e$, pero ahora caracterizada según la descripción D', de manera que $e$ ahora no satisfaga D. Querríamos que la teoría nos garantice la relación de referencia de $t$ a $e$ tanto en T como en T'. Pero, si el descriptivismo causal es correcto y T' es verdadera, entonces $t$ no puede referir a $e$ en T, porque $e$ no satisface $\mathrm{D}$, y la satisfacción de $\mathrm{D}$ es necesaria para la referencia de $t$ a $e$ en $\mathrm{T}$, independientemente de que entre en juego o no un elemento causal.

A diferencia de la primera versión del DC, en la cual el elemento causal estaba constituido por las propiedades esenciales de la entidad, en esta segunda versión puede cambiar la descripción D sin que cambie, necesariamente, el referente. Pero sigue sin ser claro cómo interviene el elemento causal para determinar la referencia. La inclusión de las propiedades estables contingentes, entonces, si bien logra evitar otros problemas, no resulta útil para lograr una teoría genuinamente híbrida para explicar la referencia de los términos teóricos.

\subsection{Las ventajas de un descriptivismo ¿causal?}

Al presentar su teoría, Psillos también presenta las ventajas explicativas de ella. El DC contaría con los siguientes puntos a su favor:

(1) Permite evitar la continuidad referencial

(2) Explica tanto el fallo como el éxito referencial de un modo no trivial.

(3) Permite una determinación adicional del referente mediante el agregado de nuevas descripciones.

(4) Deja abierta la posibilidad de re-identificar la referencia de un término, por ejemplo, cuando resulta que $x=y$ a pesar de que $\mathrm{D}(x)$ es diferente de $\mathrm{D}^{\prime}(y)$.

(5) Muestra cómo $\mathrm{D}(x)$ es capaz de rastrear la referencia del término en cuestión.

Revista de Humanidades de Valparaíso, 2021, No 17, 169-191

(c) $(i) \Theta(9)$ CC BY-NC-ND 
Creemos que, en efecto, el DC como fue expuesto cuenta con estos beneficios (a excepción del punto (3), al cual nos referiremos más adelante).Nos parece discutible, sin embargo, que estas ventajas sean una novedad del descriptivismo causal, y no rasgos presentes en cualquier teoría descriptivista.

El punto (1) transforma en desventaja la mayor ventaja de una teoría de la referencia causal. Una teoría de ese tipo sostiene que la referencia del término está dada por la entidad que causó su primer uso, uso al cual todos los hablantes que conozcan el término están vinculados a través de una cadena histórico-causal. De esta manera, un término siempre refiere a la misma entidad, permitiendo la continuidad referencial, pero también haciéndola inevitable. Una teoría descriptivista, en cambio, permite el cambio de referente de un mismo término. Si un término está asociado con una descripción y, por un cambio de teoría, pasa a estar asociado con otra descripción, entonces cambia de referente, independientemente del acto de bautismo en el cual fue introducido y de las cadenas histórico-causales que existan entre ese acto y los usos posteriores.

El punto (2) incluye dos ventajas: la de explicar el éxito referencial de modo no trivial y la de explicar el fallo referencial de un modo no trivial. La capacidad de una teoría descriptivista para hacer lo primero ya fue explicada: mientras que para una teoría causal el éxito referencial puede ser trivial, no es así para una teoría descriptivista, que atribuye un referente a un término sólo cuando la descripción asociada con él es satisfecha por una entidad. Esto nos lleva a la segunda ventaja, que es justamente el mayor desafío para una teoría causal, ya que, como dijimos, si el referente de un término es la entidad que causó su primer uso, la misma existencia del término implica la existencia del referente. La capacidad de explicar el fallo referencial es un beneficio exclusivo de una teoría descriptivista, y la razón más frecuente para preferirla a su competidora causal. Una teoría descriptivista puede explicar el fallo referencial, ya que el término puede existir y ser usado $y$, a la vez, estar asociado a una descripción que no sea satisfecha por ninguna entidad (o que sea satisfecha por más de una entidad).

El punto (3) no parece ser una ventaja de una teoría de la referencia. De hecho, no parece ser algo que una teoría de la referencia haga. Una teoría de la referencia debe ofrecer una explicación del mecanismo por el cual se determina el referente de un término. Pero, una vez que éste fue determinado, ¿qué significa dar una determinación adicional? Desde una teoría causal, el referente está determinado por la entidad que causó el primer uso del término. El agregado de nuevas descripciones no modificará el hecho de que el referente es el que es, sólo modificará, tal vez, la situación epistémica en la que se encuentran los hablantes en cuanto a esa entidad. Desde una teoría descriptivista, en cambio, el referente está determinado por la descripción asociada al término. El referente será la entidad (o clase de entidades) que cumpla con esa descripción (o conjunto de descripciones). Tampoco es claro dentro del descriptivismo cómo se daría una determinación adicional del referente mediante descripciones agregadas. Dentro de ese marco, las descripciones sí cumplen una función en la determinación del referente. Pero, si las descripciones agre- 
gadas seleccionan otras entidades, entonces no hay una determinación adicional del referente, sino que se determina otro referente para el término. Pensemos en un término $t$, asociado a un conjunto de descripciones $\mathrm{D}$ que seleccionan un conjunto de entidades $\mathrm{E}$. Si se agregan más descripciones a $\mathrm{D}$, transformándolo en $\mathrm{D}$ ', y ese conjunto de descripciones D' selecciona un subconjunto de E, al que podemos llamar E', entonces $t$ ya no refiere a las entidades que pertenecen a E, sino que refiere a las entidades de E'. El referente de $t$ no sufrió una determinación adicional, el referente de $t$ cambió: pasó de ser E a ser E'. Podría ser, también, que se agreguen descripciones pero que D' seleccione las mismas entidades que D. En ese caso, el referente de $t$ sigue siendo E. Tampoco en este caso hay una determinación adicional del referente, porque no existe tal cosa: las descripciones determinan un referente para el término al que están asociadas o no lo hacen, no hay grados de determinación del referente.

En cuanto al punto (4), la capacidad del descriptivismo para identificar el referente de dos descripciones diferentes ya fue explicada en una sección anterior, a través del ejemplo de Héspero y Fósforo. Como dijimos antes, una teoría descriptivista no prohíbe que una entidad sea seleccionada por más de una descripción. Las descripciones serán capaces de mediar la referencia entre el término y la entidad, siempre y cuando ninguna de ellas sea satisfecha por más de una entidad.

Finalmente, el punto (5) enuncia precisamente aquello que define a una teoría como descriptivista. Las descripciones son capaces de rastrear el referente porque, según el descriptivismo, la relación entre el término y el referente está mediada por la descripción asociada. Si contamos con la descripción adecuada, contaremos con un mecanismo exitoso para rastrear la entidad a la que nuestro término refiere.

La condición causal que DC menciona no es, a fin de cuentas, una condición causal que el DC exija. Ya sea porque tal condición no es genuinamente causal o porque, si lo es, no cumple ninguna función en la determinación de la referencia, resulta que el descriptivismo causal funciona exactamente como una teoría descriptivista.

El hecho de que el DC es una teoría descriptivista más se ve reflejado en sus ventajas, que no son más que las ventajas de cualquier teoría descriptivista. Y no sólo sus ventajas son las mismas que las de un descriptivismo, sino que también lo son sus dificultades. El DC es incapaz de dar cuenta de la referencia de los términos teóricos a menos que pueda dar con una descripción que tenga el alcance adecuado para mantenerse a través de diferentes teorías y, así, permitir la continuidad referencial. Hasta ahora, el descriptivista causal no ha dado con esa descripción, ya que la descripción en propiedades constitutivas de clase resulta demasiado fuerte y la descripción en propiedades estables contingentes es demasiado débil (Borge 2017). 


\subsection{Un descriptivismo causal demasiado causal}

Hasta ahora, hemos examinado el funcionamiento del DC de Psillos y hemos encontrado que, si su elemento descriptivista funciona como una teoría descriptivista lo requiere, entonces su elemento causal no cumple ningún rol en la determinación de la referencia y esta teoría no puede considerarse genuinamente híbrida.

Podría proponerse, sin embargo, otro tipo de teoría híbrida en la cual las descripciones no actúen como lo hemos descripto. El elemento descriptivista podría consistir en descripciones que no seleccionan una única entidad o que no necesitan ser conocidas por los hablantes. En ese caso, las descripciones no serían suficiente para determinar la referencia y sí sería necesario incluir un elemento causal. Tendríamos, entonces, una teoría en la cual el elemento causal sí cumple una función en la determinación de la referencia y en la que interviene, además, un elemento descriptivo.

Pero recordemos que, como mencionamos arriba, el uso de descripciones no es un problema para las teorías causales de la referencia. Tanto Putnam (1973) como Kripke (1972) admiten que las descripciones pueden tener un rol en la fijación del referente en el acto del bautismo. Putnam (1975), además, sostiene que interviene alguna clase de concepto en la introducción de un término de clase natural (como lo es, por ejemplo, el marcador "líquido" en la introducción del término "agua"). Para una teoría causal, ese elemento descriptivo no es responsable de la determinación de la referencia, por lo que no necesita seleccionar de forma única al referente ni ser conocido por todos los hablantes que usen exitosamente el término, es decir, que no necesita ser descriptivista.

La pretensión de que una teoría causal no es pura porque incluye elementos descriptivos sólo puede ser sostenida mediante una caracterización simplificada y falaz de la teoría causal de la referencia (Raatikainen 2007). Es claro que los conceptos intervienen en la relación entre hablantes y referentes, y ningún defensor de la teoría causal lo niega. Pero esos conceptos no son parte del mecanismo de fijación de la referencia. Ese mecanismo está determinado por la relación causal entre el uso del hablante y el referente. Y dicha relación es suficiente por sí misma: el referente de un término será aquel que esté vinculado causalmente con los usos de ese término, independientemente de las descripciones que cada hablante pueda asociar con él.

Una teoría causal-descriptivista en la cual el elemento descriptivo consiste en una descripción que puede ser incompleta, falsa o ignorada, no tiene nada de descriptivista. Tal teoría funciona, en última instancia, como una teoría causal pura, y no aporta nada nuevo al abordaje de la referencia de los términos teóricos. 


\section{Kitcher: modos y potencial de referencia}

Hemos argumentado que el descriptivismo causal falla en su intento de amalgamar en una cláusula única elementos descriptivistas y elementos causales para erigirse como una genuina teoría híbrida de la referencia. El resultado es que muchas de sus pretendidas ventajas son las mismas que esperaríamos encontrar en una teoría descriptivista estándar, y el aspecto causal que se introduce para salvar las desventajas del descriptivismo estándar presenta, entre varios problemas, dos fundamentales: primero, el sentido en el que el descriptivismo causal es causal no coincide ni en su naturaleza ni en sus resultados con el sentido que 'causal' tiene en las teorías causales de la referencia; segundo, incluso ignorando lo anterior, la teoría de la referencia presentada por Psillos no consigue rastrear la referencia de los términos que designan inobservables a lo largo del cambio teórico en clave realista, es decir, no cumple con la función elemental que una teoría híbrida de la referencia debería cumplir. Las razones subyacentes a dichos problemas del descriptivismo causal son diversas. Puede pensarse que una de ellas reside en lo más hondo de su estrategia: el proyecto de fundar una teoría de la referencia en la que elementos descriptivistas y causales actúan simultáneamente fijando y estabilizando la referencia bien puede estar viciado desde el principio. Amerita entonces analizar la viabilidad de una teoría híbrida en la que los elementos descriptivistas y causales no se fusionen en un único mecanismo referencial.

Ese es precisamente el caso de la propuesta de Kitcher (1993). Uno de sus rasgos más relevantes es que en ella no se postula un único mecanismo que incluye aspectos descriptivistas y causales, sino dos mecanismo diferenciados, uno de ellos descriptivista y otro causal, que se alternan fijando la referencia en usos exitosos y fallidos de un mismo término. Un segundo punto importante es que, a diferencia de lo que sucede con el DC, el aspecto causal que esta teoría incorpora coincide con la caracterización típica acuñada por la teoría de la referencia directa.

El objetivo de Kitcher es dar cuenta de cómo ciertas partes del lenguaje y prácticas correspondientes a teorías científicas abandonadas efectivamente referían, mientras que otras fracasaban en su intento de describir el mundo. El caso histórico con el que ilustra su análisis se remonta al siglo XVIII. Atiende a las consideraciones de Joseph Priestley acerca del aire desflogistizado, en el contexto de su compromiso más amplio con la teoría del flogisto. Kitcher asume, en clave realista, la intuición de que Priestley tenía muchas creencias erradas acerca de lo que realmente ocurría en sus experimentos. La teoría del flogisto es falsa, por lo que la obvia conclusión es que sus términos teóricos (v. g. 'flogisto') no refieren. Sin embargo, de ello se sigue que ninguna proposición conteniendo la expresión 'aire desflogistizado' que Priestley pudiera haber expresado tendría un contenido verdadero o aproximadamente verdadero. Que nada de lo que Priestley haya afirmado acerca del 'aire desflogistizado' nos aproxima siquiera remotamente a alguna verdad acerca del oxígeno. No obstante, el carácter continuo y acumulativo que el realismo adjudica al conocimiento científico parece entrar en tensión con esta consecuencia y

Revista de Humanidades de Valparaíso, 2021, No 17, 169-191 
dejar inexplicados el éxito predictivo y los desarrollos experimentales que hicieron ganar popularidad a la teoría del flogisto. Es por ello que Kitcher sugiere que aquella conclusión no es del todo razonable. Priestley había llevado a cabo rigurosos experimentos que le permitieron inferir diversas propiedades de su 'aire desflogistizado', y la historia de la química lo recuerda como el primero en aislar y estudiar una muestra de oxígeno. Bajo esta luz resulta poco plausible sostener que Priestley no ha dicho jamás una verdad acerca del oxígeno $(1993,100)$. La jugada que permite a Kitcher hacer justicia a ambas intuiciones descansa en una tesis central: 'aire desflogistizado', en tanto expresión tipo (type), no refiere uniformemente; sin embargo, considerada como expresión caso (token), refiere exitosamente en algunos de sus usos (mientras que otras no lo hacen). Esta distinción se relaciona estrechamente con otros dos conceptos centrales a la reconstrucción de cómo un término teórico adquiere y mantiene su referencia. El primero es el de modo de referencia, que Kitcher introduce de la siguiente manera:

Imaginemos que un hablante produce la instancia [(token)] de un término, digamos, 'la estrella de la mañana', y de ese modo refiere a un objeto. De algún modo una conexión es establecida entre los sonidos que el hablante produce y una parte de la naturaleza. Llamaré a aquello que hace que sea el caso que esa instancia refiera a ese objeto el modo de referencia de esa instancia. Para cualquier caso en el que alguien usa exitosamente una expresión para referir, podemos preguntar qué hace que sea el caso que en efecto la instancia refiera a la entidad que refiere (¿cuál es su modo de referencia? (Kitcher 1993, 76)

Cada instancia caso de una expresión tipo tiene un modo de referencia que no tiene por qué coincidir con el de otras instancias caso del mismo término tipo. Kitcher distingue tres modos de referencia: (i) bautismal, que coincide con la propuesta de las teorías causales sobre cómo se fija la referencia de un término, (ii) descriptivo, que es su contraparte descriptivista, y (iii) conformista, en el que el hablante reproduce el modo de referencia heredado de $\mathrm{su}(\mathrm{s})$ interlocutor(es). En este último caso, la referencia debe rastrearse retroactivamente en una cadena causal al momento en que fue efectivamente fijada mediante un caso de (i) o (ii). Un detalle importante: son las intenciones de los hablantes las que determinan el modo de referencia de una instancia. Si alguien fija la referencia mediante el modo descriptivo es porque tiene la "intención dominante de seleccionar algo que satisfaga una descripción particular y el referente de la instancia es cualquier cosa que satisfaga la descripción". En cambio, la referencia queda fijada bautismalmente cuando el hablante tiene la intención dominante de "seleccionar un objeto presente particular (o un conjunto de objetos, uno de cuyos miembros está presente)" (Kitcher 1993, 77).

La configuración resultante de los modos de referencia de las instancias de un término (tipo) es lo que denomina su potencial de referencia $(1993,78)$. En resumidas cuentas, la estrategia de Kitcher consiste en discriminar, dentro del potencial total de referencia de un término, aquellos modos bautismales o descriptivos en los que cada una de sus

Revista de Humanidades de Valparaíso, 2021, No 17, 169-191

(c) $(9)(9)$ CC BY-NC-ND 
instancias pretende alcanzar su referente: los primeros, por ostensión u otro mecanismo que exprese la intención relevante del agente; los segundos, por la mediación de una descripción teórica de la entidad postulada en la utilización del término con la intención relevante. Es importante notar que los modos de referencia tienen lugar en las instancias de un término, y no en el término tipo. Es en virtud de ello que Kitcher puede afirmar que aun cuando un término tipo no tenga — dado su potencial de referencia - éxito referencial, es posible que algunas instancias caso de dicho término efectivamente refieran. El término 'flogisto' y todos sus derivados, tal como nos lo informa la química moderna, no refieren a nada considerando globalmente su potencial de referencia. Sin embargo, el éxito temprano de Priestley se debe a que, al menos en algunos casos, algunas de sus instancias referían. Cuando Priestley señalaba y nombraba una muestra de lo que suponía era aire desflogistizado, al igual que cuando describía sus propiedades benéficas para la respiración, su uso de esa expresión refería en realidad al oxígeno mediante un modo de referencia bautismal. Cuando, en cambio, describía dicho gas como la sustancia liberada en la combustión, esta nueva instancia de la expresión no conseguía referir exitosamente.

La formulación de la propuesta de Kitcher presenta varios problemas. Nos detendremos en dos de ellos: en primer lugar, consideraremos el rol de las intenciones individuales como recurso para dar cuenta de la articulación de los relatos de las teorías causal y descriptivista en la noción de modo de referencia. En segundo lugar, centrándonos en la noción de potencial de referencia, argumentaremos que incluso cuando dicha articulación sea posible, eso no es suficiente para configurar una genuina teoría híbrida de la referencia.

En primer término, si bien esta posición no altera el sentido original de 'descriptivo' o 'bautismal', tal como aparecen en las teorías de la referencia correspondientes, la apelación a las intenciones individuales de los hablantes como base para la distinción de dichos modos de referencias para las instancias de uso de un término introduce una novedad que resulta distante de los fundamentos clásicos de ambas teorías. Para el descriptivismo, en la mayoría de sus variantes, el hecho de que la referencia quede fijada por una descripción se presenta como un fenómeno semántico (no psicológico), en principio ajeno a las intenciones de cada hablante en particular. En cuanto a las teorías de la referencia directa, basta recordar el lema con que Putnam pone una de las piedras fundamentales de esa tradición: los significados no están en la cabeza $(1975,227)$. La apelación a estados psicológicos individuales como medio central para fijar la referencia de un término es ajena a las dos tradiciones que la propuesta híbrida en juego pretende amalgamar. Sin embargo, incluso si se supusiera que las intuiciones u otros estados psicológicos de los hablantes en 
efecto pueden tener un rol relevante en el fenómeno de la referencia ${ }^{3}$, no es en absoluto claro que, de hecho, tengan un rol en modo que este fenómeno ocurre en la historia de la ciencia. Los defensores de la teoría del flogisto hacían inferencias que incluían instancias de expresiones como 'flogisto' o 'aire desflogistizado' alternando usos bautismales y descriptivos sin un patrón aparente que pueda relacionarse de algún modo con estados psicológicos diferenciados, mucho menos conscientes. Tal como señala Psillos (1997, 263) no hay evidencia textual en los escritos y crónicas de la época que sugieran que los defensores de la teorías del flogisto supusieron que términos como 'flogisto' fueran ambiguos de algún modo. Sus intenciones o estados psicológicos individuales, por lo tanto, no parecen resultar recursos adecuados para distinguir entre instancias que apelan a uno y otro modo de referencia, ni atendiendo al caso histórico invocado, ni revisando las teorías de la referencia en las que dichos modos de referencia se fundamentan. En el mismo sentido, no resulta claro qué tipo de criterios de identidad podrían ofrecerse para las intenciones individuales que determinan el modo conformista de la referencia.

Pero incluso ignorando este punto, hay todavía una cuestión más apremiante para esta propuesta, que reside en el corazón de sus tesis centrales. Recordemos que la noción de potencial de referencia de un término (tipo) se construye a partir de los modos de referencia, descriptivos o bautismales, de sus instancias (casos). Eso presupone que los modos de referencia pueden estar efectivamente anclados a los casos o instancias, en lugar de vinculados a la expresión tipo correspondiente. Tradicionalmente, los mecanismos por los cuales un término queda ligado referencialmente a una entidad concreta en el mundo requieren entender dicho término como una entidad abstracta, es decir, como una expresión tipo. Eso quedó de manifiesto en la presentación de las teorías descriptivistas y de la referencia directa que emprendimos más arriba. Tomemos como ejemplo un caso típico en el marco de estas últimas. Un acto de bautismo vincula una entidad concreta con un nombre. Ahora bien, ese nombre no puede ser entendido como otra entidad concreta, por ejemplo, como los sonidos proferidos por un hablante o como ciertas marcas trazadas sobre un papel. Si así fuera, sería imposible que la referencia sea transmitida causalmente de hablante en hablante. Lo que queda vinculado en ese acto de bautismo no son dos entidades concretas, sino la entidad concreta nombrada y una entidad abstracta, el término tipo. Tal como observa Psillos (1997, 260): "los casos (tokens) de las expresiones tipo no tienen, por así decirlo, propiedades semánticas autónomas, sino que las adquieren por vía de sus tipos".

No obstante, el problema es aún más profundo. No se trata simplemente de que las propiedades semánticas decantan de los tipos a los casos, sino de que el estatus mismo

\footnotetext{
${ }^{3}$ No obstante, en el caso del descriptivismo, puede pensarse que algunas de las variantes posteriores a los desarrollos de Kripke y Putnam incorporan elementos intencionalistas, sean de doble factor, teorías de referencia no directa o covariacionales (v. g. Fodor 1987). Agradecemos a un evaluador anónimo de RHV por llamar nuestra atención sobre este punto.
}

Revista de Humanidades de Valparaíso, 2021, No 17, 169-191 
de expresión caso tiene su fundamento en las expresiones tipo. Debe notarse que lo que Kitcher denomina expresión caso no es otra cosa que un objeto físico, sea un conjunto de marcas de tinta en un papel, una secuencia de sonidos, una configuración de píxeles, etc. En los campos de la filosofía del lenguaje y de la mente se han librado innumerables batallas respecto de cómo es que dichos objetos llegan a adquirir significado, pero se ha consensuado en general que, sea como fuere que eso suceda, el carácter significativo de una instancia - i.e., ¡lo que lo convierte en instancia! - está dado por su vínculo con la expresión tipo correspondiente. En el caso de los relatos descriptivistas acerca de la referencia esto resulta evidente. Si una instancia del término 'mesas' — digamos, esta misma instancia que aparece ahora ante los ojos del lector- refiere a las mesas, ello se debe a su vínculo con una expresión tipo a la que una comunidad lingüística asocia una descripción característica — V. g. tabla rasa con patas-, y no con el objeto físico que se acaba de ver e interpretar como una instancia de la expresión tipo 'mesas', ni con nuestros propósitos al ubicar allí ese objeto en lugar de otro.

La cuestión es menos transparente cuando se trata del modo causal o bautismal de referencia. Reconstruyamos brevemente el relato de Kitcher con ayuda de la siguiente notación: sea 'flogisto'-T la expresión tipo, a la que le corresponden expresiones caso que denominaremos 'flogisto'-C. Ante diferentes circunstancias, tanto teóricas como experimentales, Priestley utilizaba la expresión 'flogisto'-C, de modo tal que todas ellas correspondían a 'flogisto'-T. Al menos en algunos casos, por ejemplo cuando aludía a las propiedades benéficas que el flogisto tenía para la respiración, su uso del término 'flogisto'-C era bautismal, es decir, se ajustaba al modo en el que la teoría de la referencia directa describe la relación entre un término y su referente. Pero en este cuadro, no es en absoluto claro que, tal como Kitcher pretende, el uso de 'flogisto'-C sea efectivamente una instancia de 'flogisto'-T. Recordemos que al nominar ostensivamente una entidad con cierta expresión, la teoría causal de la referencia prescribe la formación de un lazo metafísico indisoluble entre las propiedades esenciales de esa entidad y la expresión utilizada. Pero lo que ello instituye, sin embargo, en una nueva expresión tipo a la que se vincularán todas las expresiones caso ligadas causalmente al acto bautismal por una cadena de transmisión de la referencia. Es la pertenencia a la clase de las expresiones integrantes de dicha cadena, y ninguna otra cosa, lo que determina que las expresiones caso correspondan a la expresión tipo instituida en el acto de bautismo. La mera similaridad (gráfica o sonora) entre dos expresiones caso no es suficiente para asegurar que correspondan a la misma expresión tipo, por el contrario, es posible que ambas se relacionen con dos expresiones tipo diferentes, que resulten ser homónimas. Puesto de otro modo, dos personas pueden llamarse Juana Pérez, pero eso no implica que cada una de las veces que sus nombres son mencionados se produzca una instancia de la misma expresión tipo, sino que cada uso de 'Juana Pérez' se vincula con la expresión tipo instituida en el momento en que alguien determinado fue bautizado como Juana Pérez, dependiendo de la cadena de transmisión causal a la que dicho uso corresponda. Volviendo a nuestro ejemplo, la mera similaridad 
entre varios usos de 'flogisto'-C no determina que correspondan a una y la misma expresión 'flogisto'-T, por el contrario, lo que el escenario presentado por Kitcher sugiere es que los usos exitosos de 'flogisto'-C corresponden a una expresión 'flogisto'-T diferente a la que corresponden los usos no referenciales. Una posible respuesta de Kitcher podría aferrarse al hecho de que no es la mera semejanza entre instancias, sino la intención de Priestley (algunas veces exitosa, otras veces no) de referir a siempre a la misma sustancia lo que determina el uso de una única expresión 'flogisto'-T. Pero esta réplica, dados los fundamentos de su propuesta, resultaría inadecuada, puesto que allí donde se pretenda identificar un uso causal o bautismal de 'flogisto'-C, las intenciones, propósitos o creencias de Priestley no intervendrían en absoluto en cuál es la referencia del término 'flogisto'-C, ni en cuál la expresión tipo a la que corresponde.

Una salida posible sería considerar que - refiriéndonos ahora sólo a expresiones tipo- - Priestley utilizaba en realidad dos términos diferentes - llamémoslos 'flogisto,' y 'flogisto ${ }_{2}$ - para referirse a la sustancia primordial de su teoría, uno de los cuales no denotaba ninguna entidad, mientras que el otro refería en realidad al oxígeno. Sólo cuando Priestley usaba uno de ellos, digamos 'flogisto,', conseguía dar cuenta de algún aspecto de la naturaleza. Con todo, esta eventual solución tiene algunas obvias dificultades. En primer lugar, tal como hemos señalado más arriba, nada en los registros históricos sugiere que Priestley ni ningún otro teórico del flogisto haya usado el término 'flogisto' de modo ambiguo o con matices semánticos diferentes. En segundo lugar, tanto la distinción entre 'flogisto,' y 'flogisto ${ }_{2}$ ', como el eventual resultado de la tarea de determinar cuándo Priestley usaba uno u otro término lucen como meros recursos ad hoc cuyo único propósito es salvar la continuidad referencial a lo largo del cambio teórico.

A diferencia del DC, la propuesta de Kitcher preserva los elementos casual y descriptivo propios de las teorías de la referencia que intenta articular. No obstante, hemos mostrado que esta posición presenta serias dificultades a la luz de las cuales su estatus de genuina teoría híbrida de la referencia puede ser cuestionado. Primero, el rol central que asigna a las intenciones de los hablantes es ajeno a las teorías de la referencia que pretende combinar. Segundo, la teoría no brinda un adecuado fundamento de la atribución de propiedades semánticas autónomas a las instancias de un término tipo. Tercero, dicha atribución resulta ajena a las teorías de la referencia que se busca articular y su aplicación resulta en la imposibilidad de rastrear la continuidad referencial a lo largo del cambio teórico. El diagnóstico más plausible a la luz de la postulación de propiedades semánticas autónomas para diversas instancias de un término es que se tratan en realidad de instancias de diferentes términos. En tal caso, el componente causal y el descriptivo funcionan de manera completamente independiente, desarticulando por completo la posibilidad de que esta propuesta pueda ser tomada como una genuina teoría híbrida de la referencia. 


\section{Conclusión}

Las teorías de la referencia estándar, i.e. el descriptivismo y la teoría causal de la referencia, no son capaces de cumplir, en sus formas "puras", las condiciones requeridas para una teoría de la referencia de los términos teóricos. Diversas propuestas intentan reunirlas en una teoría que recoja los puntos fuertes de ambas y excluya sus puntos débiles. En este trabajo hemos analizado críticamente dos de ellas, que ilustran estrategias diferentes para hibridar los componentes causal y descriptivo.

El descriptivismo causal propone que la referencia de los términos teóricos esté determinada por un único mecanismo que incluye tanto un elemento causal como uno descriptivista. Al analizar el funcionamiento de este mecanismo, sin embargo, encontramos que ambos elementos no pueden ser responsables, a la vez, de la determinación de la referencia. Por un lado, el elemento causal no parece ser una relación causal entre el referente y el hablante, sino una relación causal entre el referente y algún fenómeno. Es decir, que se trata de una descripción causal. Por otro lado, más allá de la naturaleza de ese elemento, Psillos describe el mecanismo de fijación y rastreo de la referencia de manera que pueda ser llevado a cabo sólo por el elemento descriptivista. En ese sentido, no se diferencia de una teoría descriptivista pura. Y no sólo eso, sino que las ventajas que el autor atribuye a su teoría son las ventajas con las que cualquier teoría descriptivista cuenta, sin incluir ningún beneficio que pueda ser atribuido al elemento causal. Advertimos, además, que si el elemento descriptivista fuera insuficiente para fijar la referencia, entonces no sería un elemento genuinamente descriptivista, y la teoría sería nada más que una teoría causal pura de la referencia. El descriptivismo causal no es más que una teoría descriptivista. Por lo tanto, cuenta con las mismas ventajas y enfrenta los mismos desafíos para explicar la referencia de los términos teóricos que cualquier descriptivismo.

La propuesta de Kitcher postula a través de las nociones de modo y potencial de referencia dos mecanismo diferenciados, uno descriptivista y otro causal, que se alternan fijando la referencia en usos exitosos y fallidos de un mismo término. De ese modo, intenta poder rastrear la referencia de los términos teóricos de acuerdo con las pretensiones realistas. Sin embargo, hemos argumentado que falla en articular una genuina teoría híbrida de la referencia en virtud de problemas respecto del rol que asigna a las intenciones de los hablantes, de su atribución de propiedades semánticas autónomas a las instancias de un término tipo y a la consecuente imposibilidad de rastrear la continuidad referencial a lo largo del cambio teórico.

En ambos casos, el intento por combinar una teoría descriptivista con una teoría causal no sólo no elimina las dificultades para explicar la referencia de los términos teóricos, sino que implica proponer mecanismos cuyo funcionamiento es, por diferentes motivos, impracticable. Si bien es claro que las teorías estándar de la referencia en sus versiones puras fallan al aplicarse a estos términos y que ambas necesitan ser modificadas, el punto de reposo del péndulo entre una y otra parece sencillamente no existir. 
Si bien estas no son las únicas propuestas en esta línea, consideramos que las dificultades presentadas ilustran un punto que puede hacerse extensivo a otras variaciones de las teorías híbridas: sea distinguiendo sus casos de aplicación en diferentes instancias de un mismo término, sea apostando por su aplicación conjunta en todas las instancias, los mecanismos causales y descriptivistas de fijación y transmisión de la referencia no parecen poder combinarse satisfactoriamente. La estabilidad que brinda el anclaje de la referencia a las propiedades esenciales de una clase no puede relativizarse parcialmente a la contingente suerte de las descripciones elaboradas por la ciencia acerca de dicha clase. Y dado que ese equilibrio entre los componentes causales y descriptivos no puede alcanzarse, la balanza termina por inclinarse por alguno de ellos.

\section{Referencias bibliográficas}

Borge, B. (2015). Realismo científico hoy: a 40 años de la formulación del Argumento del No-Milagro. Acta Scientiarum. Human and Social Sciences, 37(2), 221-233.

Borge, B. (2017). ¿Soluciona el descriptivismo causal el problema de la referencia de los términos teóricos? Ideas y Valores, 66(163), 125-151.

Borge, B. y Serebrinsky, D. (2022, en prensa) Nuevas perspectivas sobre el Realismo científico: ontología y semántica. En H. Palma y A. Gonzalez Burgos (eds.) Filosofía de las Ciencias. Nuevos debates y controversias. San Salvador de Jujuy: EDIUNJu.

Fodor, J. A. (1987). Psychosemantics: The problem of meaning in the philosophy of mind. Cambridge, MA: MIT Press.

Frege, G. (1962 [1892]). On Sense and Reference. En P. Geach y M. Black (eds.) Translations from the Philosophical Writings of Gottlob Frege, pp. 56-78. Oxford: Blackwell.

Kripke, S. (1972). Naming and Necessity. Cambridge, MA: Harvard University Press.

Kitcher, S. (1993). The Advancement of Science. New York: Oxford University Press.

Psillos, S. (1997). Kitcher on reference. International Studies in the Philosophy of Science, 11(3), 259-272.

Psillos, S. (1999). Scientific Realism: How Science Tracks Truth. London: Routledge.

Psillos, S. (2012). Causal Descriptivism and the Reference of Theoretical Terms. En Athanassios Raftopoulos and Peter Machamer (eds.), Perception, Realism, and the Problem of Reference, pp. 212-238. Cambridge: Cambridge University Press.

Putnam, H. (1973). Explanation and Reference. En G. Pearce and P. Maynard (eds.), Conceptual Change, pp. 199-221. Dordrecht: D. Reidel.

Putnam, H. (1975). The Meaning of Meaning. En Arthur Danto (Ed.) Philosophical Papers, Vol. II: Mind, Language, and Reality, pp. 215-271. Cambridge: Cambridge University Press. 
Raatikainen, P. (2007). Theories of reference and the philosophy of science. Presentado en EPSA07: 1st Conference of the European Philosophy of Science Association, Madrid, 15-17 Noviembre.

Russell, B. (1911). Knowledge by Acquaintance and Knowledge by Description. Proceedings of the Aristotelian Society, 11, 108-128.

Searle, J. (1958). Proper Names. Mind, 67(266), 166-173. 
